\title{
Metabolism and Neurogenesis
}

Marlen Knobloch* and Sebastian Jessberger*

Laboratory of Neural Plasticity, Faculty of Medicine and Science, Brain Research Institute, University of Zurich, 8057 Zurich, Switzerland

${ }^{*}$ Correspondence should be addressed to M.K. (knobloch@hifo.uzh.ch) or S.J. (jessberger@hifo.uzh.ch)

Editorial correspondence to:

Sebastian Jessberger

Brain Research Institute

University of Zurich

8057 Zurich

Switzerland

jessberger@hifo.uzh.ch 


\section{Abstract}

The generation of neurons in the developing and adult mammalian brain by neural stem/progenitor cells (NSPCs) depends on a tight control of NSPC activity and neuronal differentiation that is regulated by a plethora of intrinsic and extrinsic molecular cues. Besides well-studied morphogenic signaling pathways and transcriptional codes that govern the distinct developmental steps from the dividing NSPC to a functional neuron, a critical role of cellular metabolism to determine the functional properties of NSPCs and newborn neurons has been recently identified. Here, we review advances in our understanding of how metabolism affects NSPC behavior and subsequent neuronal differentiation and suggest how metabolism may serve as a common signal integrator to ensure life-long addition of new neurons in the mammalian brain.

\section{Introduction}

The brain is the most complex organ in mammals. The numbers of neural cells, their positioning within brain areas, the subtype specification of neurons, and the connectivity of individual neurons and subregions need to be tightly controlled to ensure the proper functioning of neural circuits [1,2]. During embryonic development dividing neural stem/progenitor cells (NSPCs) generate the vast majority of neurons that will populate the adult mammalian brain. The formation of the central nervous system has been extensively 
studied and key cellular and molecular principles have been identified that regulate the expansion of NSPCs, the induction of the generation of neurons, and subtype specification of neuronal as well as glial cells [1-3]. Notably, the process of generating neurons, called neurogenesis, does not stop with the end of embryonic and early postnatal development but continues throughout life in distinct regions, such as the hippocampal dentate gyrus (DG) and the subventricular zone (SVZ) [4]. Thus, identifying the mechanisms governing NSPC behavior is not only needed to understand the formation of the brain but is also required to understand the principles of neurogenesis that occurs throughout life in the mammalian brain. Given the complexity of the end product, it is not a surprise that each step during embryonic and adult neurogenesis is regulated by a variety of intrinsic mechanisms and cellextrinsic cues, e.g., regulated through defined transcriptional codes and key morphogenic signaling pathways $[1,5,6]$.

Until recently a core component of each cell, its metabolism, has been largely neglected for the role it may play during neurogenesis. However, it seems quite obvious that cellular metabolism determining for example the cell's energy status will be linked to NSPC activity and neuronal differentiation processes, as cell division and differentiation are associated with an increase in cell volume and biomass production and require substantial amounts of energy for DNA replication and organelle synthesis [7]. Indeed, extensive analyses of the transcriptomes of distinct NSPC stages as well as transgenesis-based gain- and loss-of-function studies indicated that distinct metabolic states play a critical role to govern developmental steps in the course of embryonic and adult neurogenesis [e.g., 6,8-13]. Here, we will 
concisely review recent evidence of how cellular metabolism affects NSPC activity and subsequent neuronal differentiation. Further, we will discuss how metabolism may serve as a molecular hub to integrate a variety of signaling pathways regulating neurogenesis during embryogenesis and in the adult mammalian brain.

\section{Metabolic control of NSPC activity}

To ease understanding, a simplified scheme of the major cellular metabolic pathways is shown in Figure 1.

\section{Lipid metabolism and NSPC activity}

Distinct lipid metabolic pathways have been known for many years as the "lipogenic phenotype" in cancer, providing proliferation and survival advantages [14]. Interestingly, similar lipid metabolic pathways are important for adult neurogenesis [12]. Proliferating adult NSPCs upregulate the production of lipids through fatty acid synthase (FASN)- dependent de novo lipogenesis, and pharmacological or genetic manipulation of this pathway is associated with a drastic reduction in proliferation and neurogenesis, suggesting a crucial role for newly formed lipids in NSPCs [12]. Further, de novo lipogenesis in NSPCs is upregulated with running, a robust enhancer of adult NSPC proliferation, showing a direct influence of a pro-neurogenic stimulus on lipid metabolism in NSPCs [15]. The chronic pharmacological inhibition of FASN abolished the beneficial effects of exercise such as increased proliferation and cognitive enhancement [15], supporting the importance of lipid metabolism to control NSPC activity. 
The amount of de novo lipogenesis also influences quiescence behavior as the production of new lipids is reduced in quiescent adult NSPCs through the action of a specifically expressed protein called Spot14 regulating the levels of Malonyl-CoA, one of the substrates for FASN [12]. The dynamic response of Spot14-positive NSPCs to pro- and anti-neurogenic stimuli further suggest that NSPCs can alter their lipid metabolism upon extrinsic signals [16], in line with increased FASN activity upon running [15]. However, how exactly these extrinsic signals translate into a metabolic change remains to be elucidated. Though it is not yet clear why proliferating adult NSPCs are so dependent on newly produced lipids, it is likely that a large amount is used for new membrane production required upon proliferation and differentiation, as suggested by radioactive tracing experiments[12].

Interestingly, it has recently been suggested that NSPCs might not only use lipids as building blocks for membranes but also as an alternative energy source to glucose. Increased fatty acid oxidation (FAO), the breakdown of lipids, was found to be high in adult NSPCs in the SVZ and pharmacological inhibition of FAO resulted in reduced proliferation [17]. Extending the role of FAO for NSPCs, it was shown that the rate of FAO controls adult NSPC activity and serves as a counter-regulatory pathway to de novo lipogenesis (Knobloch et al. submitted 2016). Quiescent NSPCs have high levels of FAO that are reduced upon entry into a proliferative state. NSPC quiescence is accompanied by high levels of Spot 14 and by concomitant low levels of malonyl-CoA, which are reversed both upon activation. Intriguingly, besides serving as substrate for de novo lipogenesis, high levels of malonyl-CoA also inhibit FAO. Thus, malonyl-CoA and Spot14 might control these two opposed 
lipid metabolic pathways and determine whether NSPCs remain quiescent or proliferate (Knobloch et al. submitted 2016). Furthermore, a recent report linked the clinical association of FAO deficits with neuropsychiatric diseases to a dysregulation of NSPC activity during development [18]. Inhibition of FAO resulted in a reduced NSPC pool, which was due to increased differentiation and reduced self-renewal of NSPCs, suggesting that FAO is indeed crucial for the maintenance of NSPCs [18]. How exactly FAO functions to maintain NSPCs remains to be determined.

Lipid metabolism also seems to be important for the interaction between the niche and NSPCs: two recent papers showed that the accumulation of lipids in so-called lipid droplets in niche cells might directly influence NSPC behavior [19,20]. In Drosophila, lipid droplets in niche cells protect both glia and neuroblasts by providing a "safe" storage for polyunsaturated fatty acids during oxidative stress and thus limit damage inflicted by peroxidation reactions [19]. Whether similar mechanisms exist in the mammalian neurogenic niches is not known. However, an excess of lipid droplets in the ependymal cells lining the ventricular zone, part of the niche in the mammalian SVZ, have been recently associated with Alzheimer disease pathology [20]. In an Alzheimer mouse model, this lipid accumulation leads to decreased NSPC proliferation, which could be mimicked in wildtype mice by a local increase and subsequent accumulation of lipids, suggesting that perturbed lipid metabolism in disease might be directly influencing NSPC behavior [20]. Taken together, there is now strong evidence pointing towards an important regulatory role of lipid metabolism in NSPCs that might open new avenues for manipulating NSPC behavior. 


\section{Mitochondrial activity and NSPC behavior}

Another emerging view on metabolic changes between stem cells and progeny suggests that mitochondrial mass/activity and oxidative phosphorylation increase with lineage progression whereas glycolytic activity is rather a stem cell feature [21]. This also seems to hold true for NSPCs and their differentiating neuronal progeny (see below), as recent publications suggest [22]. Low levels of oxygen, i.e., hypoxia, has been known for a long time to promote NSPC maintenance and proliferation both in vitro and in vivo, however, the underlying mechanisms have remained poorly understood [23]. Two recent studies have now addressed how hypoxia influences NSPC metabolism. The first study addressed the reliance of embryonic and adult NSPCs on oxidative versus glycolytic metabolism, using pharmacological inhibition and alteration of substrate availability [24]. Indeed, NSPCs were highly tolerant to hypoxia, but inhibition of glycolytic pathways, even when another oxidizable substrate was provided, greatly impaired their survival [24]. Supporting a role of glycolytic versus oxidative metabolism for NSPC behavior, an elegant study connected the ingrowth of blood vessels into the developing cortex and the resulting increase in oxygen availability with the well-known switch of NSPC expansion to differentiation during brain development [25]. Using a genetic perturbation of vessel formation, the authors showed that hypoxia due to the absence of ingrowing blood vessels caused the NSPC pool to expand whereas neurogenesis was dramatically reduced. Hyperoxygenation of the pregnant mothers restored tissue oxygen in the embryos despite the lack of blood vessels and rescued the phenotype. 
Hif1 $\square \square \square$ was highly upregulated and functional studies using genetic knockout and overexpression approaches showed its regulatory role in this system, mainly by upregulating glycolysis [25].

Although mitochondrial metabolism seems to be lower in NSPCs than in their neuronal progeny, mitochondria still contribute to NSPC activity regulation, for instance through $\mathrm{FAO}$ as outlined above. A certain level of oxidative metabolism might even be necessary to prevent oncologic transformation of NSPCs, as has been recently suggested: inhibition of mitochondrial metabolism in NSPCs led to a switch towards more glycolysis with higher proliferation and less inducible differentiation [26]. Genetic impairment of mitochondrial function increased tumor-forming capacity of NSPCs when transplanted into the brain of recipient mice, suggesting that a tight metabolic control might be crucial to prevent uncontrollable growth [26]. A reduction in mitochondria has also been linked to age related changes in NSPCs, accompanied by general alterations in metabolism [27]; however, the detailed mechanisms and consequences of lowered mitochondrial content require further studies, including in vivo analyses. Interestingly, a recent study showed that repletion of oxidized nicotinamide adenine dinucleotide (NAD+) appears to be efficient to improve mitochondrial function in aged somatic stem cells and extend lifespan [28].

Reactive oxygen species (ROS) production and defense affect NSPC activity The generation of ROS is closely linked to mitochondrial metabolism. It is widely known that ROS can be toxic side products of the electron transfer chain, causing damage to macromolecules such as proteins, lipids and DNA. 
Thus, antioxidant mechanisms antagonizing the negative effects of ROS are important for cellular health. Defects in these defense mechanisms have been associated with aging and disease, whereas successful reduction of ROS, for instance through caloric restriction, has been put forward as one underlying cause for an increase in longevity [29]. It is becoming evident, however, that a certain amount of ROS is important for signaling and may be involved in regulating stem cell behavior [30-33]. Thus, understanding the regulation of ROS and the maintenance of the cellular redox potential is also relevant for the field of stem cell biology where direct or indirect roles for ROS regulating NSPC activity have been described [30,34]. Several key metabolic and lifespan regulators such as forkhead Box $\mathrm{O}$ (FoxO) transcription factors, sirtuins, as well as mammalian target of rapamycin (mTOR), have been linked to ROS production, ROS defense and redox potential and have been recently shown to be important for NSPCs. Ablation of FoxOs results in an initial increase in brain size followed by reduced neurogenesis and a reduction of the NSPC pool, suggesting an important role of FoxOs in controlling selfrenewal $[31,32]$. ROS levels were increased upon FoxO ablation and gene expression signatures of FoxO knockout vs. wildtype NSPCs point towards regulation of oxygen and glucose metabolism $[31,32]$. Indeed, a recent study focused on the metabolic alterations upon FoxO ablation and identified glycolysis and glutamine metabolism to be downregulated [35]. This downregulation resulted in increased oxidative stress and compromised the proliferative potential of NSPCs, most likely because both downregulated metabolic pathways normally contribute to the anti-oxidant defense program of a cell [35]. Although these studies show the importance of FoxOs for 
NSPCs and link it to metabolic changes, further studies are needed to better understand these effects.

Similarly, sirtuins have been shown to be critical for neurogenesis. Sirtuins are protein deacetylases with manifold actions and implications in various processes such as life span, inflammation and cancer [36]. Their deacetylating activity on histones has direct transcriptional consequences and their dependence on NAD+ as a co-enzyme makes them perfect sensors of the redox state of a cell. Thus far, most of the publications studying sirtuins in the context of neurogenesis have shown effects on differentiation (see below). However, two recent publications have provided evidence that sirtuins might also regulate NSPC self-renewal and proliferation $[37,38]$. Extracellular glucose levels regulated NSPC proliferation via a coordinated mechanism of Hes-1 expression, repressed by sirtuin 1 and activated by CREB, directly linking nutrient availability to NSPC behavior [38]. Remarkably, and despite the fact that many laboratories traditionally use high glucose medium to culture NSPCs, low glucose had beneficial effects on the self-renewal of NSPCs [38], indicating that the classic culture conditions may need to be adapted with increasing knowledge of metabolic requirements of NSPCs.

Nutrient availability also activates mTOR signaling [39]. Both inhibition and hyperactivation of mTOR have recently been shown to affect NSPC quiescence, proliferation and differentiation, suggesting a tight balance of optimal mTOR activity [40-42]. Interestingly, mTOR activity diminishes with age in NSPCs and might be relevant for the age-associated decline in neurogenesis, supported by the finding that mTOR inhibition in NSPCs in vitro resulted in a reversible quiescence-like phenotype [41]. Indeed, it was shown 
that stimulation of mTOR signaling in aged mice increases NSPC proliferation [43]. In summary there is clear evidence that metabolism indeed regulates NSPC activity; however, detailed and comprehensive metabolic analyses will be required to fully understand how the different key players affect the metabolic state of NSPCs.

\section{Metabolic mechanisms regulating neuronal differentiation and maturation}

Many metabolic pathways that alter NSPC behavior ultimately also affect the generation of newborn neurons. Thus, it is difficult to identify specific pathways that only regulate neuronal differentiation and maturation but do not affect NSPCs per se. As outlined above, an increase in mitochondrial metabolism is emerging as a key feature associated with stem cell differentiation $[21,44]$. In an elegant study in Drosophila, a steroid-hormonemediated metabolic switch from glycolysis to oxidative phosphorylation was shown to trigger cell cycle exit and terminal differentiation of NSPCs during metamorphosis [45]. Inhibition of oxidative phosphorylation by genetically targeting various components of the mitochondrial electron transport chain prevented this differentiation and extended the life span of the neuroblasts [45], suggesting a direct regulation of NSPC differentiation in Drosophila via oxidative phosphorylation. In the mammalian system, an extensive increase of mitochondrial mass in adult newborn neurons compared to NSPCs has been described [8]. Upon exercise, the increase in mitochondria was even more profound, and virus-mediated genetic manipulation of mitochondrial mass either inhibited neurogenesis (when mitochondria generation was knocked 
down) or further enhanced neurogenesis (when mitochondria generation was augmented), providing a functional link between mitochondrial metabolism and neuronal maturation [8]. Similarly, genetic mitochondrial damage in NSPCs had a detrimental effect on the generation of neurons and oligodendrocytes during development, with only minor effects on the NSPC pool [46].

Interestingly, a large amount of adult newborn neurons die during maturation, a well-described but poorly understood phenomenon [47]. Given the large increase in mitochondria during the differentiation process, a concomitant increase in ROS production may contribute to increased cell death. Indeed, increased oxidative stress was reported to occur as a side effect of NSPC proliferation/differentiation [48], although the direct consequences were not elaborated. A recent publication in the field of cellular reprogramming showed that direct conversion of fibroblasts or astrocytes into neurons was accompanied by a significant increase in oxidative stress, which led to massive cell death and inefficient generation of neurons [49]. Antioxidative treatment significantly lowered cell death and resulted in a better yield of reprogrammed neurons. Strikingly, activating the vitamin D pathway, which is known for its anti-oxidative action, more than doubled direct reprogramming in vivo [49], emphasizing the important role of oxidative phosphorylation and the redox state for differentiation.

Support for the dependence of proper differentiation on an optimal redox state also comes from several recent publications studying sirtuins in the context of neurogenesis. As described above, sirtuins are good redox state sensors, as they depend on NAD+, the abundance of which is directly 
reflecting the oxidative state of a cell. Activation of sirtuin 1 repressed neuronal differentiation whereas knockdown led to an increase in neurogenesis in vitro and in vivo [50,51], proposing that oxidative challenges that often accompany aging and disease might directly suppress neurogenesis via activation of sirtuin 1. However, two recent studies rather suggest that such regulation may be more complex: while one study show that inducible genetic inactivation of sirtuin 1 in adult mice increases oligodendrocyte progenitors rather than neurons and enhances proliferation of NSPCs [53], others found that the ablation of the NAD+ producing enzyme Nampt as well as knockout of sirtuins led to reduced oligodendrocyte production and reduced NSPC proliferation [52]. These divergent results are most likely caused by the complex and manifold actions of sirtuins. Future detailed characterization of the metabolic changes upon manipulation of sirtuins might shed light onto these findings.

In addition, certain lipid metabolic pathways might also directly influence neuronal maturation and differentiation. As outlined above, several recent studies have uncovered the importance of lipid metabolism for NSPC quiescence and proliferation and also showed altered neurogenesis, yet most likely through alteration of NSPC behavior rather than through a direct influence on differentiation. Cholesterol metabolism however might be a pathway of specific importance for developing neurons [54]. The specific ablation of endogenous cholesterol production in NSPCs led to massive apoptosis of newborn neurons during development, resulting in death at birth, whereas NSPCs were able to compensate the lack of cholesterol by 
increased uptake from the circulation [54]. The reason for this difference in coping with a lack of cholesterol remains to be elucidated.

\section{Conclusions}

Substantial progress has been made in the last decades to decipher the mechanisms underlying the formation of the brain and the life-long addition of neurons by NSPCs. In addition to key transcriptional regulators and signaling pathways it has been recently discovered that cell metabolism plays a role in controlling NSPC activity and subsequent neuronal differentiation. Future studies will have to determine the exact metabolic switches (or shifts) occurring with distinct cellular states of NSPCs (e.g., quiescence vs. activation). Furthermore, it will be of interest to determine how fast metabolic adaptations occur and if for example metabolic differences may already occur for each prospective daughter cell during asymmetric cell division of NSPCs.

Given its central role in determining the cellular state, cell metabolism may serve as a signal integrator that "translates" a variety of signals into an integrated metabolic response that may affect cellular physiology and behavior on multiple levels (e.g., by changing energy state, fuel source, biomass production, and epigenetics). Thus, studying the interplay between transcriptional programs, morphogenic signaling, and its down- or upstream regulation of the metabolic state may substantially improve our understanding of how NSPCs orchestrate the construction of the brain. 


\section{Acknowledgements}

We thank Darcie L. Moore for critical comments on the manuscript. Our laboratory is supported by the Swiss National Science Foundation, EMBO Young Investigator program, Théodore Ott foundation, Novartis foundation, and the European Research Council. M.K. was supported by the JanggenPöhn foundation. The authors declare no competing financial interests.

\section{Figure legends}

Figure 1: A simplified scheme of the major cellular metabolic pathways. Glucose is taken up and metabolized into pyruvate in a process called glycolysis, with a relatively small amount of energy equivalents, adenosine triphosphate (ATP) and reduced nicotinamide adenine dinucleotide (NADH), generated. Pyruvate can be either fermented into lactate, which is subsequently secreted, or can be shuttled into the mitochondria and used in the tricarboxylic acid (TCA) cycle to generate NADH and reduced flavin adenine dinucleotide $(\mathrm{FADH})$ for energy production. In the mitochondrial respiratory chain, the NADH and FADH generated during the TCA cycle are used in a complex process called oxidative phosphorylation (OXPHOS), requiring oxygen $\left(\mathrm{O}_{2}\right)$ and resulting in the generation of energy in the form of ATP. As a side product, reactive oxygen species (ROS) can be generated 
during OXPHOS. NADH and FADH are also generated in large amounts by the breakdown of fatty acids in a process termed fatty acid oxidation (FAO), occurring in mitochondria as well as in peroxisomes (not shown in the scheme). The resulting acetyl-CoA can be fuelled into the TCA cycle for further energy production and as a carbon source or can be exported from mitochondria via citrate for other use. For instance, acetyl-CoA is one of the building blocks for the generation of new lipids (lipogenesis) in a process involving fatty acid synthase (FASN), yielding palmitate, which can be subsequently used to generate more complex fatty acids. The reduced nicotinamide adenine dinucleotide phosphate (NADPH) required for lipogenesis can be generated during the pentose phosphate pathway (PPP), a metabolic pathway parallel to glycolysis.

Figure 2: Schematic representation of the major metabolic pathways in NSPCs and their neuronal progeny.

Shown are the major changes in metabolic pathways occurring in quiescent and proliferating NSPCs as well as in immature neurons in the adult dentate gyrus. Please refer to the text for details.

\section{References}

- Of special interest

$\bullet \quad$ Of outstanding interest 
1. Taverna E, Gotz M, Huttner WB: The cell biology of neurogenesis: toward an understanding of the development and evolution of the neocortex. Annu Rev Cell Dev Biol 2014, 30:465-502.

2. Rowitch $\mathrm{DH}$, Kriegstein AR: Developmental genetics of vertebrate glialcell specification. Nature 2010, 468:214-222.

3. Fernandez V, Llinares-Benadero C, Borrell V: Cerebral cortex expansion and folding: what have we learned? EMBO J 2016, 35:1021-1044.

4. Bond AM, Ming GL, Song H: Adult Mammalian Neural Stem Cells and Neurogenesis: Five Decades Later. Cell Stem Cell 2015, 17:385-395.

5. Pollen AA, Nowakowski TJ, Chen J, Retallack H, Sandoval-Espinosa C, Nicholas CR, Shuga J, Liu SJ, Oldham MC, Diaz A, et al.: Molecular identity of human outer radial glia during cortical development. Cell 2015, 163:55-67.

6. Telley L, Govindan S, Prados J, Stevant I, Nef S, Dermitzakis E, Dayer A, Jabaudon D: Sequential transcriptional waves direct the differentiation of newborn neurons in the mouse neocortex. Science 2016, 351:1443-1446.

- $\quad$ 7. Ito K, Suda T: Metabolic requirements for the maintenance of selfrenewing stem cells. Nat Rev Mol Cell Biol 2014, 15:243-256.

A thorough review covering the metabolic regulation of stem cell activity.

8. Steib K, Schaffner I, Jagasia R, Ebert B, Lie DC: Mitochondria modify exercise-induced development of stem cell-derived neurons in the adult brain. J Neurosci 2014, 34:6624-6633.

9. Llorens-Bobadilla E, Zhao S, Baser A, Saiz-Castro G, Zwadlo K, MartinVillalba A: Single-Cell Transcriptomics Reveals a Population of Dormant Neural Stem Cells that Become Activated upon Brain Injury. Cell Stem Cell 2015, 17:329-340.

10. Beckervordersandforth R, Tripathi P, Ninkovic J, Bayam E, Lepier A, Stempfhuber B, Kirchhoff F, Hirrlinger J, Haslinger A, Lie DC, et al.: In vivo fate mapping and expression analysis reveals molecular hallmarks of prospectively isolated adult neural stem cells. Cell Stem Cell 2010, 7:744-758. 
11. Bracko O, Singer T, Aigner S, Knobloch M, Winner B, Ray J, Clemenson GD, Jr., Suh H, Couillard-Despres S, Aigner L, et al.: Gene expression profiling of neural stem cells and their neuronal progeny reveals IGF2 as a regulator of adult hippocampal neurogenesis. The Journal of neuroscience : the official journal of the Society for Neuroscience 2012, 32:3376-3387.

- 12. Knobloch M, Braun SM, Zurkirchen L, von Schoultz C, Zamboni N, Arauzo-Bravo MJ, Kovacs WJ, Karalay O, Suter U, Machado RA, et al.: Metabolic control of adult neural stem cell activity by Fasndependent lipogenesis. Nature 2013, 493:226-230.

This study showed that adult neural stem cells depend on a specialized lipid metabolism for proper proliferation.

- 13. Shin J, Berg DA, Zhu Y, Shin JY, Song J, Bonaguidi MA, Enikolopov G, Nauen DW, Christian KM, Ming GL, et al.: Single-Cell RNA-Seq with Waterfall Reveals Molecular Cascades underlying Adult Neurogenesis. Cell Stem Cell 2015, 17:360-372.

Extensive ressource paper that delineates the molecular signature of neural stem cell states in the adult hippocampus.

14. Menendez JA, Lupu R: Fatty acid synthase and the lipogenic phenotype in cancer pathogenesis. Nat Rev Cancer 2007, 7:763777.

15. Chorna NE, Santos-Soto IJ, Carballeira NM, Morales JL, de la Nuez J, Catala-Valentin A, Chornyy AP, Vazquez-Montes A, De Ortiz SP: Fatty acid synthase as a factor required for exercise-induced cognitive enhancement and dentate gyrus cellular proliferation. PLOS One 2013, 8:e77845.

16. Knobloch M, von Schoultz C, Zurkirchen L, Braun SM, Vidmar M, Jessberger S: SPOT14-positive neural stem/progenitor cells in the hippocampus respond dynamically to neurogenic regulators. Stem Cell Reports 2014, 3:735-742.

17. Stoll EA, Makin R, Sweet IR, Trevelyan AJ, Miwa S, Horner PJ, Turnbull DM: Neural Stem Cells in the Adult Subventricular Zone Oxidize 
Fatty Acids to Produce Energy and Support Neurogenic Activity. Stem Cells 2015, 33:2306-2319.

18. Xie Z, Jones A, Deeney JT, Hur SK, Bankaitis VA: Inborn Errors of Long-Chain Fatty Acid beta-Oxidation Link Neural Stem Cell SelfRenewal to Autism. Cell Rep 2016, 14:991-999.

19. Bailey AP, Koster G, Guillermier C, Hirst EM, MacRae JI, Lechene CP, Postle AD, Gould AP: Antioxidant Role for Lipid Droplets in a Stem Cell Niche of Drosophila. Cell 2015, 163:340-353.

20. Hamilton LK, Dufresne M, Joppe SE, Petryszyn S, Aumont A, Calon F, Barnabe-Heider F, Furtos A, Parent M, Chaurand P, et al.: Aberrant Lipid Metabolism in the Forebrain Niche Suppresses Adult Neural Stem Cell Proliferation in an Animal Model of Alzheimer's Disease. Cell Stem Cell 2015, 17:397-411.

21. Wanet A, Arnould T, Najimi M, Renard P: Connecting Mitochondria, Metabolism, and Stem Cell Fate. Stem Cells Dev 2015, 24:19571971.

22. Homem CC, Repic M, Knoblich JA: Proliferation control in neural stem and progenitor cells. Nat Rev Neurosci 2015, 16:647-659.

23. Simon MC, Keith $B$ : The role of oxygen availability in embryonic development and stem cell function. Nat Rev Mol Cell Biol 2008, 9:285-296.

24. Candelario KM, Shuttleworth CW, Cunningham LA: Neural stem/progenitor cells display a low requirement for oxidative metabolism independent of hypoxia inducible factor-1alpha expression. J Neurochem 2013, 125:420-429.

- 25. Lange C, Turrero Garcia M, Decimo I, Bifari F, Eelen G, Quaegebeur A, Boon R, Zhao H, Boeckx B, Chang J, et al.: Relief of hypoxia by angiogenesis promotes neural stem cell differentiation by targeting glycolysis. EMBO J 2016, 35:924-941.

This study elegantly links angiogenesis to neural stem cell proliferation in the developing forebrain with levels of glycolysis.

26. Bartesaghi S, Graziano V, Galavotti S, Henriquez NV, Betts J, Saxena J, Minieri V, A D, Karlsson A, Martins LM, et al.: Inhibition of oxidative 
metabolism leads to p53 genetic inactivation and transformation in neural stem cells. Proc Natl Acad Sci U S A 2015, 112:1059-1064.

27. Stoll EA, Cheung W, Mikheev AM, Sweet IR, Bielas JH, Zhang J, Rostomily $\mathrm{RC}$, Horner PJ: Aging neural progenitor cells have decreased mitochondrial content and lower oxidative metabolism. J Biol Chem 2011, 286:38592-38601.

28. Zhang H, Ryu D, Wu Y, Gariani K, Wang X, Luan P, D'Amico D, Ropelle $E R$, Lutolf MP, Aebersold R, et al.: NAD+ repletion improves mitochondrial and stem cell function and enhances life span in mice. Science 2016.

29. Lopez-Lluch G, Navas P: Calorie restriction as an intervention in ageing. J Physiol 2016, 594:2043-2060.

30. Le Belle JE, Orozco NM, Paucar AA, Saxe JP, Mottahedeh J, Pyle AD, $\mathrm{Wu} \mathrm{H}$, Kornblum $\mathrm{HI}$ : Proliferative neural stem cells have high endogenous ROS levels that regulate self-renewal and neurogenesis in a PI3K/Akt-dependant manner. Cell Stem Cell 2011, 8:59-71.

31. Renault VM, Thekkat PU, Hoang KL, White JL, Brady CA, Kenzelmann Broz D, Venturelli OS, Johnson TM, Oskoui PR, Xuan Z, et al.: The pro-longevity gene $\mathrm{FoxO} 3$ is a direct target of the p53 tumor suppressor. Oncogene 2011, 30:3207-3221.

32. Paik JH, Ding Z, Narurkar R, Ramkissoon S, Muller F, Kamoun WS, Chae SS, Zheng $\mathrm{H}$, Ying $\mathrm{H}$, Mahoney $\mathrm{J}$, et al.: FoxOs cooperatively regulate diverse pathways governing neural stem cell homeostasis. Cell Stem Cell 2009, 5:540-553.

33. Bigarella CL, Liang R, Ghaffari S: Stem cells and the impact of ROS signaling. Development 2014, 141:4206-4218.

- 34. Khacho M, Clark A, Svoboda DS, Azzi J, MacLaurin JG, Meghaizel C, Sesaki H, Lagace DC, Germain M, Harper ME, et al.: Mitochondrial Dynamics Impacts Stem Cell Identity and Fate Decisions by Regulating a Nuclear Transcriptional Program. Cell Stem Cell 2016. This study shows how shifts in mitochondrial metabolism affect stem cell identity and differentiation in the developing forebrain. 
35. Yeo $\mathrm{H}$, Lyssiotis $\mathrm{CA}$, Zhang $\mathrm{Y}$, Ying $\mathrm{H}$, Asara JM, Cantley LC, Paik JH: FoxO3 coordinates metabolic pathways to maintain redox balance in neural stem cells. EMBO J 2013, 32:2589-2602.

36. Houtkooper $\mathrm{RH}$, Pirinen $\mathrm{E}$, Auwerx $\mathrm{J}$ : Sirtuins as regulators of metabolism and healthspan. Nat Rev Mol Cell Biol 2012, 13:225-238.

37. Ma CY, Yao MJ, Zhai QW, Jiao JW, Yuan XB, Poo MM: SIRT1 suppresses self-renewal of adult hippocampal neural stem cells. Development 2014, 141:4697-4709.

38. Fusco S, Leone L, Barbati SA, Samengo D, Piacentini R, Maulucci G, Toietta G, Spinelli M, McBurney M, Pani G, et al.: A CREB-Sirt1-Hes1 Circuitry Mediates Neural Stem Cell Response to Glucose Availability. Cell Rep 2016, 14:1195-1205.

39. Jewell JL, Guan KL: Nutrient signaling to $m T O R$ and cell growth. Trends Biochem Sci 2013, 38:233-242.

40. Magri L, Cambiaghi M, Cominelli M, Alfaro-Cervello C, Cursi M, Pala M, Bulfone A, Garcia-Verdugo JM, Leocani L, Minicucci F, et al.: Sustained activation of mTOR pathway in embryonic neural stem cells leads to development of tuberous sclerosis complexassociated lesions. Cell Stem Cell 2011, 9:447-462.

41. Paliouras GN, Hamilton LK, Aumont A, Joppe SE, Barnabe-Heider F, Fernandes $\mathrm{KJ}$ : Mammalian target of rapamycin signaling is a key regulator of the transit-amplifying progenitor pool in the adult and aging forebrain. The Journal of neuroscience : the official journal of the Society for Neuroscience 2012, 32:15012-15026.

42. Magri L, Galli R: mTOR signaling in neural stem cells: from basic biology to disease. Cell Mol Life Sci 2013, 70:2887-2898.

43. Romine J, Gao X, Xu XM, So KF, Chen J: The proliferation of amplifying neural progenitor cells is impaired in the aging brain and restored by the mTOR pathway activation. Neurobiol Aging 2015, 36:1716-1726.

44. Xu X, Duan S, Yi F, Ocampo A, Liu GH, Izpisua Belmonte JC: Mitochondrial regulation in pluripotent stem cells. Cell Metab 2013, 18:325-332. 
- 45. Homem CC, Steinmann V, Burkard TR, Jais A, Esterbauer H, Knoblich JA: Ecdysone and mediator change energy metabolism to terminate proliferation in Drosophila neural stem cells. Cell 2014, 158:874-888.

This study represents one of the pioneering studies linking metabolism to neural stem cell activity.

46. Diaz-Castro B, Pardal R, Garcia-Flores P, Sobrino V, Duran R, Piruat Jl, Lopez-Barneo J: Resistance of glia-like central and peripheral neural stem cells to genetically induced mitochondrial dysfunction--differential effects on neurogenesis. EMBO Rep 2015, 16:1511-1519.

47. Kempermann G, Gast D, Kronenberg G, Yamaguchi M, Gage FH: Early determination and long-term persistence of adult-generated new neurons in the hippocampus of mice. Development 2003, 130:391399.

48. Walton NM, Shin R, Tajinda K, Heusner CL, Kogan JH, Miyake S, Chen Q, Tamura $\mathrm{K}$, Matsumoto $\mathrm{M}$ : Adult neurogenesis transiently generates oxidative stress. PLoS One 2012, 7:e35264.

- 49. Gascon S, Murenu E, Masserdotti G, Ortega F, Russo GL, Petrik D, Deshpande A, Heinrich C, Karow M, Robertson SP, et al.: Identification and Successful Negotiation of a Metabolic Checkpoint in Direct Neuronal Reprogramming. Cell Stem Cell 2016, 18:396-409.

This study identified a crucial step of metabolic adaptations in the context of cellular reprogramming.

50. Prozorovski T, Schulze-Topphoff U, Glumm R, Baumgart J, Schroter F, Ninnemann O, Siegert E, Bendix I, Brustle O, Nitsch R, et al.: Sirt1 contributes critically to the redox-dependent fate of neural progenitors. Nat Cell Biol 2008, 10:385-394.

51. Saharan S, Jhaveri DJ, Bartlett PF: SIRT1 regulates the neurogenic potential of neural precursors in the adult subventricular zone and hippocampus. J Neurosci Res 2013, 91:642-659. 
52. Stein LR, Imai S: Specific ablation of Nampt in adult neural stem cells recapitulates their functional defects during aging. EMBO J 2014, 33:1321-1340.

53. Rafalski VA, Ho PP, Brett JO, Ucar D, Dugas JC, Pollina EA, Chow LM, Ibrahim A, Baker SJ, Barres BA, et al.: Expansion of oligodendrocyte progenitor cells following SIRT1 inactivation in the adult brain. Nature cell biology 2013.

- 54. Saito K, Dubreuil V, Arai Y, Wilsch-Brauninger M, Schwudke D, Saher G, Miyata T, Breier G, Thiele C, Shevchenko A, et al.: Ablation of cholesterol biosynthesis in neural stem cells increases their VEGF expression and angiogenesis but causes neuron apoptosis. Proc Natl Acad Sci U S A 2009, 106:8350-8355.

This study was one of the first studies to link metabolic processes (i.e., cholesterol biosynthesis) to neural stem cell behavior in the developing forebrain. 

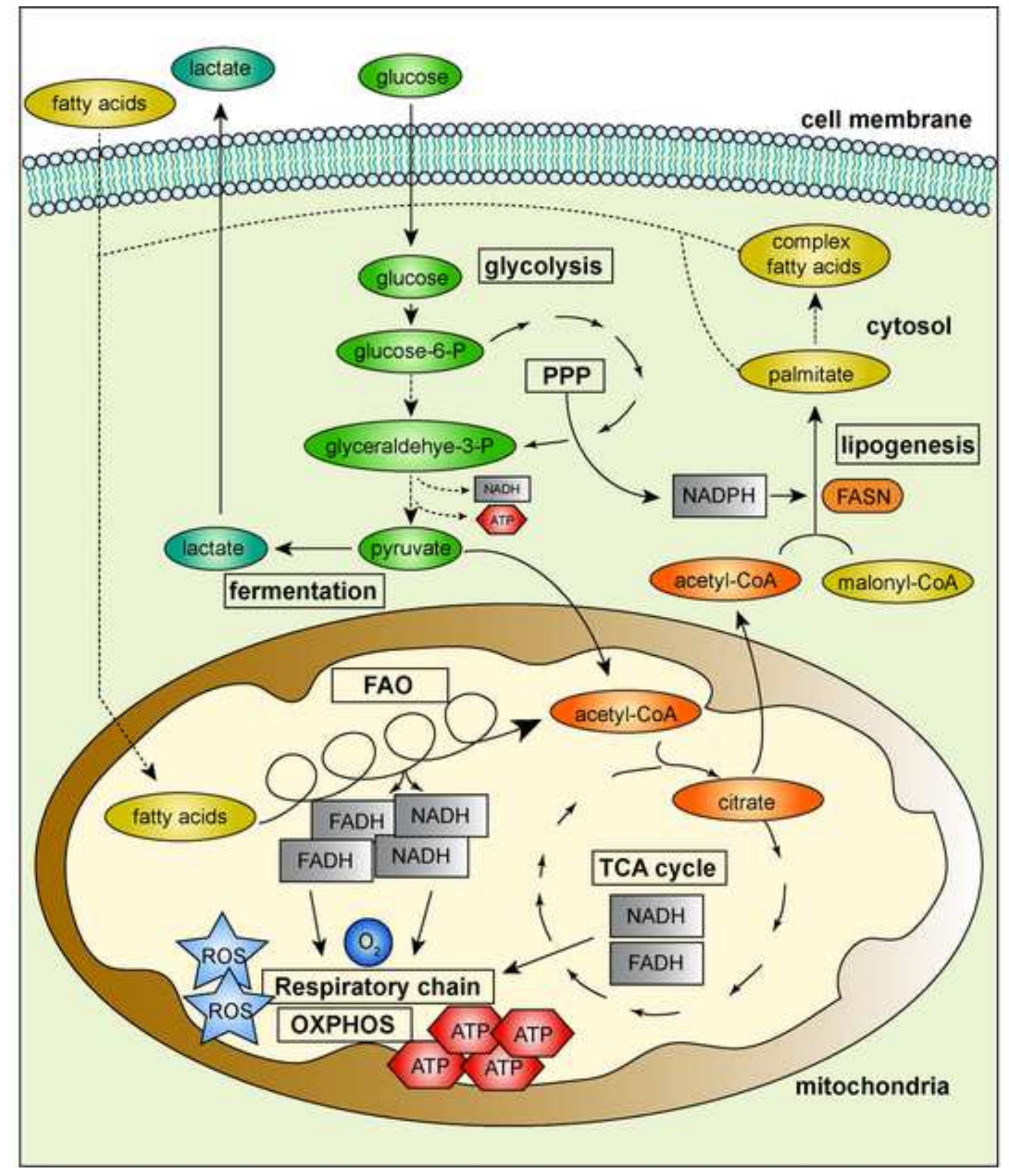


\section{quiescent NSPC \\ (radial glia-like cell)}

proliferating NSPCs

immature neurons

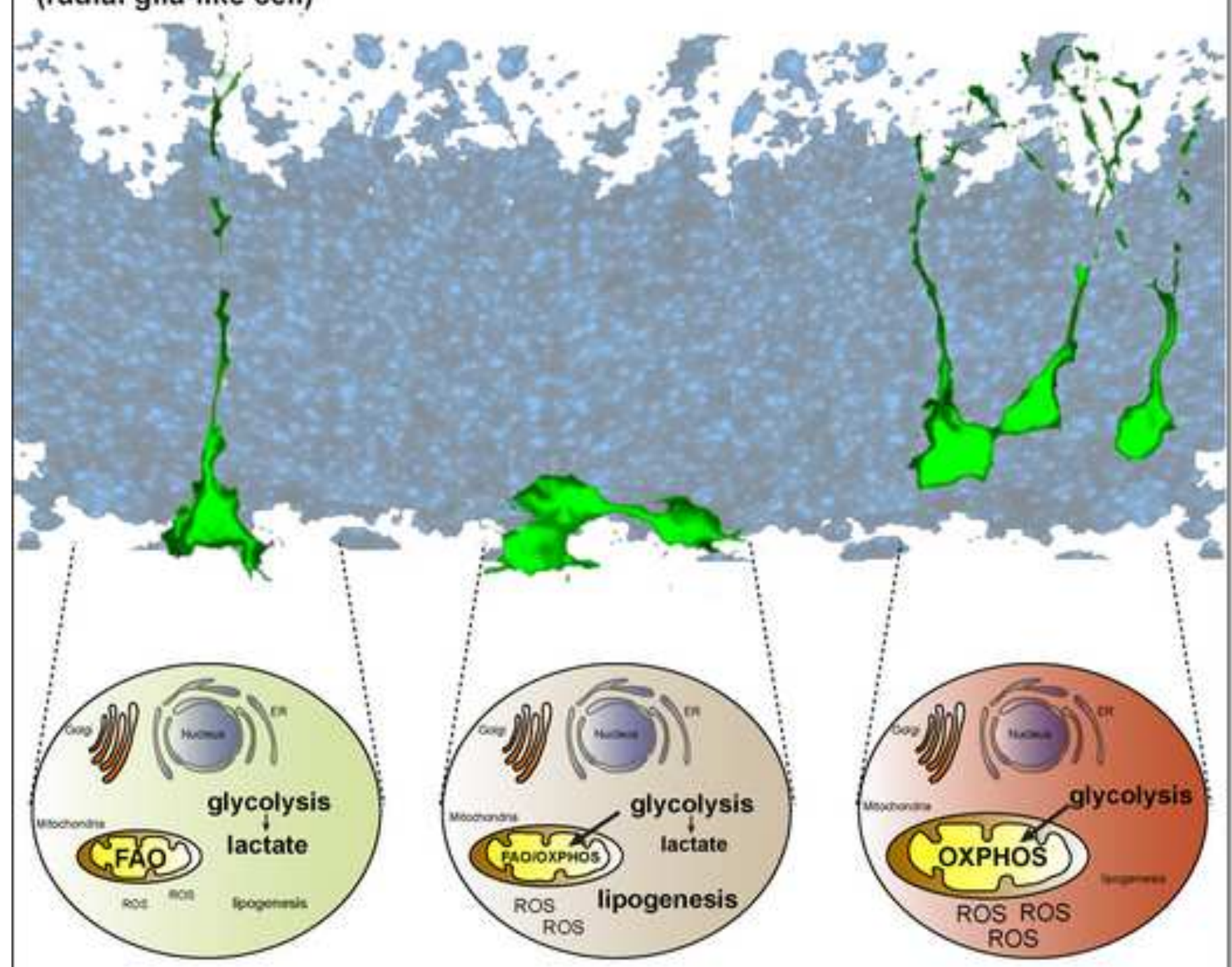

Glycolysis

(lactate)

OXPHOS

FAO

De novo

lipogenesis

ROS defense

ROS generation 